\title{
Chaos, Poverty, and Parenting: Predictors of Early Language Development
}

\author{
Lynne Vernon-Feagans, Patricia Garrett-Peters, Mike Willoughby, Roger Mills-Koonce, and \\ The Family Life Project Key Investigators \\ The University of North Carolina at Chapel Hill
}

\begin{abstract}
Studies have shown that distal family risk factors like poverty and maternal education are strongly related to children's early language development. Yet, few studies have examined these risk factors in combination with more proximal day-to-day experiences of children that might be critical to understanding variation in early language. Young children's exposure to a chronically chaotic household may be one critical experience that is related to poorer language, beyond the contribution of SES and other demographic variables. In addition, it is not clear whether parenting might mediate the relationship between chaos and language. The purpose of this study was to understand how multiple indicators of chaos over children's first three years of life, in a representative sample of children living in low wealth rural communities, were related to child expressive and receptive language at 36 months. Factor analysis of 10 chaos indicators over five time periods suggested two factors that were named household disorganization and instability. Results suggested that after accounting for thirteen covariates like maternal education and poverty, one of two chaos composites (household disorganization) accounted for significant variance in receptive and expressive language. Parenting partially mediated this relationship although household disorganization continued to account for unique variance in predicting early language.
\end{abstract}

Many household and family characteristics have been linked to poorer language development in children. Distal risk characteristics, including young maternal age, family size, poverty, and especially low maternal education have been linked to poorer language development in young children in a variety of research studies (Brody \& Flor, 1998; BrooksGunn \& Duncan, 1997; Hoff, 2003; Pan, Rowe, Spier, \& Tamis-Lemonda, 2004;

Westerlund \& Lagerberg, 2007). Many of these studies suggest that parenting mediates or partially mediates the relationship between risk and early language. Parents who were less sensitive, engaged, and verbally stimulating in interactions with their young children were more likely to be poor, less educated, and know less about parenting, and, in turn, had children with poorer language skills (Bradley \& Corwyn, 2005; Brody \& Flor, 1997; Hart \& Risley, 1995; NICHD Early Childcare Network, 2000; Raviv, Kessenick, \& Morrison, 2004).

Although these studies have documented that family poverty and other related risk characteristics are related to poorer parenting and later compromised child language, few

\section{(C) 2011 Elsevier Inc. All rights reserved}

Please address correspondence to: Lynne Vernon-Feagans (lynnevf@email.unc.edu), 301K Peabody Hall, \#3500, University of North Carolina, Chapel Hill, North Carolina 27599.

Publisher's Disclaimer: This is a PDF file of an unedited manuscript that has been accepted for publication. As a service to our customers we are providing this early version of the manuscript. The manuscript will undergo copyediting, typesetting, and review of the resulting proof before it is published in its final citable form. Please note that during the production process errors may be discovered which could affect the content, and all legal disclaimers that apply to the journal pertain. 
studies have addressed other home characteristics that might be related to children's poorer language. A construct that may tap an important stressful experience of children in these low-wealth communities is household chaos. According to Bronfenbrenner and Evans (2000), the household chaos construct can be described as "systems of frenetic activity, lack of structure, unpredictability in everyday activities, and high levels of ambient stimulation" (p. 121). Although this definition has been useful, there has been a need for a more conceptual and operational definition that encompasses the research done in this area. Among the contributors to a new edited volume on chaos (Evans \& Wachs, 2010), there seemed to be some consensus on two important constructs within the definition of chaos: turbulence/instability and disorder. Instability/turbulence was related to changes in settings and relationships in the home and the unpredictability of routines. Disorder, on the other hand, included "high levels of noise, excessive crowding, clutter, and lack of structure" (Sameroff, 2010, p. 258). Although instability and disorder are useful in the current study as the core constructs involved in defining chaos, most of the previous literature reviewed here referred to particular indicators of chaos.

The literature on chaos and child development has included a variety of specific features of household chaos in examining links to poorer parenting and child outcomes. These chaos indicators cut across the instability and disorder constructs. For instance, ambient noise in the home/neighborhood, TV watching in the home, household crowding, and disorganized family routines have been used to index disorganization, while household moves, number of people moving in and out of the home, and changes in parent figures in the home have been used to index instability (Adam, 2004; Evans, Maxwell, \& Hart, 1999; Johnson, Martin, Brooks-Gunn, \& Petrill, 2008; Matheny, Wachs, Ludwig, \& Phillips, 1995). These previous studies have used a parent report of chaos or particular indicators of chaos at one point in time to examine the relationship between chaos and children's outcomes. The purpose of the current study was to add to this literature on family chaos by examining whether objective measures of chaos over time could be represented by the molar constructs of disorganization and instability that were discussed as central to defining chaos (Evans \& Wachs, 2010). We were also interested in examining whether the accumulation of chaos in early childhood might be related to early child language in a representative sample of children living in lowwealth rural communities. In addition, we were interested in whether observed poorer parenting might be a partial mediator of the relationship between chaos and poorer early language.

\section{Chaos and Rural Low-Wealth Communities}

Over the last 30 years, there have been dramatic changes in the lives of families who live in rural low-wealth communities. For instance, jobs are no longer strongly linked to farming but have moved to the service sector where there are more irregular and non-standard work hours as well as lower wages (Lichter \& Jensen, 2002). These jobs are generally not close to where the families live so many parents must commute long distances to work and childcare. There has also been outmigration of young upwardly mobile adults from rural communities, leaving behind a less-educated and more at-risk population of childbearing age (Duncan, 1999; Lichter \& Jensen, 2002; O'Hare \& Johnson, 2004; Vernon-Feagans et al., in press). One of the consequences of these shifts in jobs and people has been an increase in child poverty and an increasing gap between child poverty in urban areas relative to non-urban areas. Over half the children in rural areas live in families whose incomes are below $200 \%$ of the federal poverty threshold. In comparison, only $37 \%$ of children in urban areas live in families whose incomes are below $200 \%$ of the poverty threshold (Rivers, 2005). This increase in poverty, irregular jobs, and the distance between work and services may have created the context for the experience of greater chaos in the lives of children. Greater economic insecurity may lead families to move frequently from one household to another in 
order to sustain the family living together and may also require adding more people to the household to increase or sustain the economic viability of the family. Demands for childcare at irregular hours and jobs that require long commutes also may add to the family's moves and add more people in the household to care for young children. These economic strategies have been documented in ethnographic studies of working class and low-income families (Duncan, 1999; Edin \& Lein, 1997; Shipler, 2004; Ward \& Turner, 2005). For instance, in a study of 75 low-income families over a four-year period, Roy, Tubbs, and Burton (2004) found that family adaptations to the demands of daily life were associated with more chaos, including higher household density (i.e., \# of rooms per person), more people in the household, and more changes in the household members. These changes due to economic insecurity have been shown to be exacerbated by changes in maternal partners that can alter the economics and emotional climate in the family through partner entrances and departures (Lichter \& Jensen, 2002). Overall, it has been shown that these chaos indicators appear to be increasing more in low-income families than middle-income families because poverty is associated with more instability of families, more nonstandard work hours, and less access to reliable transportation, childcare, etc. (Evans, Gonnella, Marcynyszyn, Gentile, \& Salpeker, 2005; Evans \& Wachs, 2010) and may be increasing even more in rural lowwealth families who must deal with the stress of geographic isolation with less access to key resources like transportation and long distances to work and childcare (O'Hare, 2009).

\section{Chaos and Early Language Development}

Early language development, especially early word learning, has been shown to be faster and more efficient when children are engaged in joint-attention activities with their mothers or other caregivers (Tomasello \& Farrar, 1986) and where caregivers are responsive to the attention and vocalizations of their young children (Tamis-LeMonda, Bornstein, KathanaKalman, Baumwell, \& Cyphers, 1998; Tomasello \& Todd, 1983). Adult language that helps the child to understand the relationships between spoken words and what they represent in the environment, coupled with sustained conversational dialogue between adult and child, promotes better word learning and language development (Arterberry, Midgett, Putnick, \& Bornstein, 2007; Brooks \& Meltzoff, 2008; Watt, Wetherby, \& Shumway, 2006).

Parents with lower levels of education have been shown to be less responsive to their children's language and to provide a less optimal environment for word learning and grammatical development (Hoff, 2008; Raviv et al., 2004). In addition, there is evidence that chaotic family environments also provide children with less optimal environments for language development. In general, this may mean that parents are just less sensitive and effective because of chaos but both Matheny et al. (1995) and Evans, Maxwell, and Hart (1998) have argued that household chaos could directly influence children's development, especially early cognitive and language development, by overwhelming children with too much stimulation. As a consequence, both articles speculated that children may cope with this overstimulation by blocking out and withdrawing from the overstimulation in the home. For instance, in a home with lots of background noise and many people going in and out of the house, a young child might not be able to process the language that is directed to him/her because of the many ambient distractions. Rather than try to persist and concentrate on the language directed toward the child, the child might turn away and avert their eyes from the overstimulation. The child might play by himself/herself and/or begin an activity that blocks out the stimulation, such as pounding a hammer or singing to himself/herself. This withdrawal would likely diminish children's ability to engage in joint-attentional activities and other parent/child interactions that promote language development. There is support for this overstimulation hypothesis in some of the chaos literature that has examined the relationship of ambient noise and overcrowding to language and literacy. Maxwell and Evans (2000) found that a single indicator of chaos, exposure to chronic noise in the 
neighborhood, was negatively related to children's preschool language development, while Evans et al. (1998) found that residential crowding was negatively related to children's language, even after controlling for SES. In further studies, household and school density (crowding) were negatively linked to poorer language/literacy and academic performance in older children (Evans, 2006; Evans, Kliewer, \& Martin, 1991; Evans, Lepore, Shejwal, \& Palsane, 1998; Maxwell, 2003). These studies of particular indicators of crowding/noise that are often associated with disorganization at one point may be linked to poorer language in children.

Unpredictability of the environment has also been hypothesized to be central to the construct of chaos (Bronfenbrenner \& Evans, 2000). This unpredictability has been operationalized to be a function of the disorganization found in some homes (Wachs, 1987; Wachs, 1989;

Wachs \& Chan, 1986). Wachs hypothesized that the lack of predictability in family routines interfered with the children's ability to extract the rules of discourse and may have affected parents' ability to interact effectively with their children. In these initial studies, environmental disorganization/unpredictability measures included such indicators as regularity in the child's schedule, the amount of time the TV was on, and the number of visitors and people in the home. The Wach studies found that those indicators of unpredictability/disorganization added significant variance beyond demographic characteristics in predicting decreased toddler mastery and language.

Other studies have tried to examine a variety of chaos constructs by using a short parent report measure of chaos that indexed many of the dimensions of chaos just mentioned; including crowding, confusion, household traffic, and ambient noise, some of which index disorganization and some instability. This questionnaire called the Confusion, Hubbub, and Order Scale (CHAOS) (Matheny et al., 1995) was not developed from a particular theoretical framework but was a more empirical scale of chaos indicators that has been used in numerous studies over the last 15 years. In the recent studies of mostly middle-income children, using this scale or parts of the scale, chaos accounted for relatively small amounts of variance in predicting language outcomes in young children (Hart, Petrill, Deater-Decker, \& Thompson, 2007; Johnson et al., 2008; Petrill, Pike, Price, \& Plomin, 2004). For instance, Petrill et al. (2004) found that SES and CHAOS were associated with a statistically significant proportion of the variance in parent-reported child language at 3 and 4 years of age, beyond that accounted for by genetic, nonshared environmental, and unidentified shared environmental influences. SES and CHAOS accounted for between $1 \%$ and $5 \%$ of the total variance and both SES and the CHAOS questionnaire partially mediated the shared environmental variance in parental reports of children's reported verbal skills at ages 3 and 4. A recent twin study composed of mostly middle- to upper-middle-class families (Johnson et al., 2008) examined the relationship among some items from the CHAOS scale, the home literacy environment, and children's expressive vocabulary during kindergarten and first grade. Factor analysis suggested two chaos factors (household order and household quiet). This study found that household order, some home literacy factors, and maternal reading ability were predictive of the child's expressive vocabulary.

\section{Chaos, Parenting, and Language Development}

The link between accumulated chaos experiences and early language has been hypothesized to be mediated by less effective parenting in many of the studies just discussed, even though almost all of these studies did not have measures that could examine this three-part link (Evans \& Wachs, 2010; Evans, 2006; Wachs, 1987). Bronfenbrenner and Evans (2000) hypothesized that chaos might disrupt the important proximal processes between parent and child, especially in families who were under stress from poverty and other factors.

Researchers have argued that this disruption in parenting might be related to the tension that 
chaos created in the home, leading to parental insensitivity and a lower motivation to actively engage with their children (Corapci \& Wachs, 2002; Evans et al., 1999; Johnson et al., 2008; Matheny et al., 1995). There has been some previous research that has linked chaos to poorer parenting. Matheny et al. (1995) demonstrated that greater chaos, as measured by the parent report CHAOS scale, was associated with more parental verbal interference, less giving of objects, and more ignoring of the child's attempts to communicate. Corapci and Wachs (2002) found that the CHAOS scale (the noise and crowding factors) was related to less responsive and stimulating parenting. This was supported by a more recent study (Coldwell, Pike, \& Dunn, 2006) that found links between household chaos and parenting, with household chaos predictive of school-age children's problem behavior over and above parenting. In addition, household chaos played a moderating role between parenting and children's behavior by exacerbating the effect of poorer-quality parenting on children's behavior. The only study to date that has actually examined the three-part link among aspects of chaos, early parenting, and child language was a study that reexamined the Hart and Risley (1995) data with the addition of a home crowding factor (Evans et al., 1999). This study found that household density (a dimension of chaos) predicted poorer maternal language input to their children and, in turn, less complex language by their children, even after controlling for SES. Parental responsiveness partially mediated this relationship. Although these studies did not examine the causal links between chaos and parenting, these authors argue that chaos may create stress that can distract parents from the task of parenting and may interfere with their ability to deliver high-quality language input and sensitive caregiving with young children.

\section{Summary and Hypotheses}

The studies reviewed here have suggested that accumulated chaos, either measured by a parent report scale (CHAOS) or by individual indicators of chaos at one point in time, is related to poorer early child language. A few studies have found that chaos is related to parenting, using the CHAOS questionnaire, and only one study suggested that an indicator of chaos (crowding) is related to poorer child language partially through its relationship to parenting. The present study adds to the literature on chaos by:1) creating a more objective and comprehensive set of chaos indicators that are not dependent on parent report of chaos, 2) developing a cumulative index of chaos that represents children's early experience of chaos over a three-year period, 3) adding a host of family and child covariates, unlike previous studies of chaos, to help make the case that chaos is important beyond demographic and other family and child variables, 4) using a representative sample of children from lowwealth communities so that the findings are more generalizable, and 5) examining whether observed parenting practices over three years might partially mediate the relationship between chaos and early language, after controlling for important covariates. From the literature reviewed, we predicted that accumulated chaos from multiple indicators over the first three years of life would be associated with poorer child language, beyond important demographic, maternal, and child variables. We also predicted that parenting would partially mediate the relationship between chaos and child language.

\section{Method}

\section{Sample and Design}

The Family Life Project (FLP) was designed to study young children and their families who lived in two of the four major geographical areas of the United States with high poverty rates (Dill \& Myers, 2008). Specifically, three counties in Eastern North Carolina and three counties in Central Pennsylvania were selected to be indicative of the Black South and Appalachia, respectively. The FLP adopted a developmental epidemiological design in which sampling procedures were employed to recruit a representative sample of 1,292 
children whose mothers resided in one of the six counties at the time of the child s birth. In addition, low-income families in both states and African American families in North Carolina were over-sampled to ensure adequate power for dynamic and longitudinal analyses of families at elevated psychosocial risk (African American families were not oversampled in Pennsylvania because the target communities were at least $95 \%$ non-African American).

At both sites, recruitment occurred seven days per week over the 12-month recruitment period spanning September 15, 2003 through September 14, 2004 using a standardized script and screening protocol. The coverage rate was over $90 \%$ for all births that occurred to women in these counties in that one year period. In Pennsylvania, families were recruited in person from three hospitals. These three hospitals represented a weighted probability sample (hospitals were sampled proportional to size within county) of seven hospitals that delivered babies in the three target Pennsylvania counties. Pennsylvania hospitals were sampled because the number of babies born in all seven target hospitals far exceeded the number needed for purposes of the design. In North Carolina, in-person recruitment occurred in all three of the hospitals that delivered babies in the target counties. Phone recruitment also occurred for families who resided in target counties but delivered in non-target county hospitals. These families were located through systematic searches of the birth records located in the county courthouses of nearby counties.

FLP recruiters identified 5,471 (59\% NC, 41\% PA) women who gave birth to a child in the 12 -month period. A total of 1,515 (28\%) of all identified families were determined to be ineligible for participation for three primary reasons. These were: not speaking English as the primary language in the home, residence in a non-target county, and intent to move within three years. This strategy may have eliminated some high-risk families. Of the 2,691 eligible families who agreed to be considered, 1,571 (58\%) families were randomly selected to participate using the sampling fractions that were continually updated from our data center. Of those families selected to participate in the study, 1,292 (82\%) families completed a home visit at 2 months of child age, at which point they were formally enrolled in the study.

The current study tested whether the cumulative experience of household chaos across the first three years of life was associated with child language outcomes at age 3 . As such, the sample was limited to the $N=1,123$ (of 1,292 total) families who participated in the 36month home visit. Families who participated in the 36 -month visit $(N=1,123)$ did not differ from those who did not participate in this visit $(N=169)$ with respect to state of residence (38.5\% vs. $40.4 \%$ residing in Pennsylvania, $p=.63)$, race of the child $(39.6 \%$ Vs. $42.9 \%$ African American, $p=.42$ ), being recruited in the low-income stratum (78.7\% vs. $77.4 \%$ poor, $p=.70)$, or being a teenage mother $(5.3 \%$ vs. $6.4 \%, p=.61)$. Among the families who participated in the 36-month visit, $98 \%$ participated in four or five of the five possible data collection time points (i.e., when target children were approximately 2, 6, 15, 24, and 36 months of age). Hence, there was appreciable longitudinal data from which to derive cumulative measures of household income, parenting, and chaos.

Demographic characteristics of the sample are summarized in Table 1. We provide both weighted and unweighted descriptives of the sample to demonstrate very few differences between the unweighted and weighted descriptive statistics resulting from both the oversampling of low income, and in NC, African American participants, as well as the larger population of children residing in PA versus NC counties.

Nearly all (99\%) of the primary caregivers (PC) were female. This was almost always the biological mother. We use the name primary caregiver to refer to the person who lives with 
the child and cares for the child. If the biological mother resided in the home, she was always considered the primary caregiver. Forty one percent of primary caregivers were African American (AA). On average, mothers were 28 years old when target children were born, although they were on average 21.6 years of age when they had their first child. The children in this study were 37.1 months old when child outcomes were measured.

Approximately half (49\%) of the children were female. There was good variation in PC education with the average years of education being $13(\mathrm{SD}=2)$. Sixteen percent of PCs had less than a high-school education, $16 \%$ had a 4-year college degree, and the remaining had some intermediate level of education (e.g., highest education equal to HS/GED degree; HS/ GED + professional training; Associate Degree). Over half (54\%) of all PCs were married and employed (57\%). On both the literacy screener and the depression inventory, mothers were below the national standardization sample means of 50 and 100, respectively. Finally, the mean income-to-needs ratio for the household was 1.9 (an income to needs ratio of 1.0 corresponds to the federal poverty threshold for that household size).

\section{Procedures}

At 2 months of age, children and their families participated in a single home visit. At the 2month visit, one research assistant used a laptop computer and entered information from the mother on demographics of household members, mother and partner employment, childcare arrangements, and other key variables. Two research assistants visited children and their families twice when the children were 6,24, and 36 months of age, and once when the child was 15 months of age, for a total of seven home visits. During the home visits, the research assistants conducted interviews with the mother and administered questionnaires, conducted child assessments, and videotaped interactions between children and adults (mother and secondary caregiver, if present).

\section{Measures}

Covariates-Thirteen control variables were used in the analyses. They included: target child age (TC age), child gender (TC gender), child temperamental distress (TC temperament distress), prenatal exposure to alcohol or illicit drug use (TC PEAD), the number of different childcare settings at each home visit (TC CC settings), primary caregiver age at $1^{\text {st }}$ birth ( $\mathrm{PC} 1^{\text {st }}$ birth), primary caregiver education (PC education in years), primary caregiver married (PC married), primary caregiver employment history (PC employment), primary caregiver depression (PC depression), primary caregiver literacy (PC literacy screen), household income/needs ratio (HH income/needs), and geographic isolation (HH geo isolation). These variables were collected at the home visits throughout the child's first three years of life and are described below.

Primary caregivers reported years of education, marital status, and birth of their first child at the 36-month visit. The target child's age was calculated from date of birth and gender was reported by the mother at the child's birth. At each of the five timepoints, primary caregivers also reported whether or not they were working and the number of childcare settings used for the target child at each home visit. Employment history was represented by the percent of home visits in which primary caregivers reported working. The number of childcare settings was represented by the average number of childcare settings attended by the child at each of the five home visit time points.

In the context of completing the pregnancy and delivery module of the Missouri Assessment of Genetics Interview for Children (MAGIC; Reich, Todd, Joyner, Neuman, \& Heath, 2003) at the 2-month home visit, mothers reported on the presence, frequency, and amount of alcohol and illicit drug (marijuana, cocaine, other 'hard' drugs) use during their pregnancy. 
Reich and colleagues reported good short and long-term reliability for self-reports of maternal smoking, alcohol, and drug use during pregnancy (Reich et al., 2003).

Primary caregivers also reported on child temperamental distress at 6 months using the Infant Behavior Questionnaire (IBQ-R) (Gartstein \& Rothbart, 2003). Primary caregivers completed questions on a seven-point Likert scale ranging from never (1) to always (7) to rate the frequency with which the child exhibited a variety of behaviors over the previous two weeks. A composite of child distress was formed using the distress to novelty (16 items) and the distress to limitations (16 items) subscales (see also Garrett-Peters et al., 2008; Hane, Fox, Polak-Toste, Ghera, \& Guner, 2006). Internal consistency for these two subscales has been reported for children in 3-6, 6-9, and 9-12 month age groups. Internal consistency for distress to limitations was $.81, .83, .82$, respectively, and for distress to novelty, internal consistency was $.90, .89$, and .87 , respectively (Gartstein \& Rothbart, 2003).

Primary caregivers' self-reported depressive symptoms were obtained using the Brief Symptoms Inventory (BSI-18, Derogatis, 1993) averaged across all time points at which the measure was collected (i.e., 2, 6, 15, and 24 months). The BSI-18 is a short and highly sensitive screening index containing eighteen items that are divided evenly across three dimensions: somatization, depression, and anxiety, and also produces a global severity index, which includes all items. Only the global severity index was used in the present study. Using a 5-point scale (ranging from $0=$ not at all to $4=$ extremely), respondents were asked to indicate the degree to which particular problems have distressed or bothered them during the past seven days. Internal consistency for the global severity index was .89 in a community based sample of 1,134 (age range $=20-69$ ) (Derogatis, 2000).

Primary caregiver literacy was obtained through the administration of the K-FAST (Kaufman Functional Academic Skills test: Reading Skills Subscale, Kaufman \& Kaufman, 1994) at the 2-month home visit. The K-FAST evaluates how well people understand written words and signs applied to everyday situations and in life tasks that involve reading competence. Internal consistency of the K-FAST has been determined by split-half estimates for subtest and composite scores in multiple samples that reflected the intended age range. Within this method, the mean reliability score across samples for the Reading Skills Subscale was .90 (range $=.86-.95)$. Test-retest reliability was obtained from a sample of 116 individuals ranging in age from 15 to 91 who performed the K-FAST twice within an interval of 6 to 94 days (mean $=33$ days). Within this method, the reliability coefficient for the Reading Skills Subscale was .88 (Kaufman \& Kaufman, 1994).

Regarding household income, the FLP adopted the approach taken by Hanson, McLanahan, and Thomson (1997) and based household income on anyone who resides in the household, not simply those people related by blood, marriage, or adoption. Individuals were considered to be co-residents if they spent three or more nights per week in the child's household. Using this information, the total annual household income was divided by the federal poverty threshold for a family of that size and composition (thresholds vary based on number of adults and children) to create the income/needs ratio. For this data, the income/needs ratio was calculated using the 2004 poverty threshold values.

A measure of geographic isolation for each family was developed using Global Positioning System (GPS) technology. This measure was the best indicator of the family's isolation from important resources that might differentiate them from more urban families. The GPS units measured the longitude and latitude for the family residence at each home visit. These were used to compute the physical distance between the family residence and 10 different community services: the nearest elementary school, high school, supermarket, county seat, 
doctors' office (any type), freeway on-ramp, library, public park, gas station, and fire station. A single summary score was computed as the mean of the 10 distances and was log transformed to reduce skew in its distribution (see also Burchinal et al., 2008).

Household Chaos-Ten cumulative indicators of household chaos were derived from data collected at home visits when target children were approximately 2, 6, 15, 24, and 36 months old. Six indicators were based on data that were collected at all five possible home visit periods (i.e., at 2, 6, 15, 24, and 36-month visits). Thus from the data at each time point, we were able to construct changes from one time point to the next for each of these variables. They included: (1) the total number of times the child moved physically to another residence, (2) the total number of changes in the primary caregiver (usually involved change in primary responsibility for child from mother to other adult), (3) the total number of changes in the secondary caregiver (either primary caregiver partner or primary caregiver grandmother), (4) the total number of different people in the household, (5) the total number of times household members moved into or out of the household, and (6) report of the average number of hours that the TV was on each day (This was a simple average of the number of hours the TV was reported to be on at each separate visit). A seventh indicator, average household density, was created using data that were collected at four home visits (i.e., 2, 6, 24, and 36 months). At each visit, the number of rooms in the home was divided by the number of people residing in the home to create a time-specific household density score. This item reflected the average density across these three time points. The eighth, ninth, and tenth indicators were consensus ratings by the two research assistants who completed the initial home visit at each time point. These indicators and the consensus procedure were selected from the post-visit Inventory used in the Fast Track intervention study (Dodge, Pettit, \& Bates, 1994) at the 2, 6, 24, and 36-month home visits that captured the disorganization in the household. These included the following three items: (1) home visit preparation by the household $(0=$ can't rate, $1=$ surprise/difficulty, $2=$ aware, but unprepared, $3=$ aware/ready, $4=$ good hosts), (2) the cleanliness of the household ( $0=$ can't rate, $1=$ very dirty, $2=$ slightly dirty, $3=$ messy, $4=$ clean), and (3) the neighborhood noise level around the home ( $0=$ can't rate, $1=$ very quiet, $2=$ average, $3=$ noisy, $4=$ very noisy). Scores of " 0 " on these indicators were treated as missing in the analyses.

Positive and Negative Parenting-Primary caregiver behaviors during play interactions with their children were assessed at the 6,15, 24, and 36-month home visits. At 6 and 15 months of age, primary caregiver behaviors were observed during a free-play interaction with toys and games. At the 24 and 36-month home visits, primary caregivers and their children were seated at a table and were asked to complete a set of three puzzles of increasing difficulty. In this task, primary caregivers were instructed to provide assistance to their child as they felt necessary. Both the free-play and the puzzle tasks lasted 10 minutes and were recorded using a DVD camcorder for later coding. Interactions were coded by independent coders who were unaware of the study's hypotheses. Seven subscales were used to evaluate primary caregiver behavior during the mother-child interaction session. The following qualitative ratings have been used in previous studies to assess the quality of parent-child interaction (NICHD Study of Early Child Care, 1999) and include: sensitivity, detachment/disengagement, positive regard, intrusiveness, animation, stimulation of development, and negative regard. Coders rated each of these areas on a 7-point Likert scale ranging from not at all characteristic (1) to highly characteristic (7). Approximately $30 \%$ of the parent codes were double-coded, which means that the final scores were reached by consensus between two coders. Each coding pair maintained an inter-rater reliability rating of .80 or above on all subscales. A Positive Parenting composite was created by summing the scale scores for sensitivity, positive regard, stimulation of development, animation, and detachment/disengagement (reverse-scored). A Negative Parenting composite was created 
by summing the scale scores for intrusiveness and negative regard for the child. For both the Positive and Negative Parenting composite, the intra-class correlation for coders ranged from .85 to .98 across the four assessment points. Given repeated measures of parenting behaviors across multiple time points (i.e., $6,15,24$, and 36 months), the mean of each composite was used in analyses to reflect the cumulative effect of positive and negative parenting.

Child Receptive and Expressive Language-At 36 months of age, the children were given a receptive and expressive language test. These tests not only tapped two different modes of language but also tapped different language skills. The Receptive Vocabulary subscale from the Wechsler Primary Preschool Inventory (WPPSI-III; Wechsler, 2003) was administered to the all children in the home. This subtest required the children to point to the picture that represented the word spoken by the administrator. There is extensive validity data on the WPPSI-III in the 2003 manual. The scores derived from the WPPSI-III correlate well with the WPPSI, WISC-R, Stanford Binet (4th ed.), and McCarthy Scales (correlations between WPPSI-R FSIQs and other test composites range from .74 to .90). The reliability coefficient of the WPPSI-III subtests range from .83 to .95 . The reliability coefficients for the composite scales ranged from .89 to .96 . Test-retest reliabilities for a mean interval of 26 days for the 2:6 to 3:11 year old group Verbal, Performance, Full, and General Language scores were $.90, .84, .92$, and .92 respectively. A licensed clinical psychologist developed procedures and standards for certification and trained all home visitors on administration and scoring. Additional training regarding administration and certification was provided to two senior staff members who served as master administrators and certifiers at each site. Certification procedures involved observing proficiency in all aspects of administration and scoring by either live observation or video-recorded administrations with 36 month olds. The master administrators observed and scored a minimum of two administrations before determining if a home visitor had met the certification criteria.

The Expressive Communication subscale of the Preschool Language Scale-4 (PLS-4; Zimmerman, Steiner, \& Pond, 2002) was used to evaluate children's Expressive Language at the 36-month visit. The Expressive Communication subscale is used to determine how well the child communicates with others. The tasks on this subscale measure verbal development and social communication. The child is asked to name common objects, use concepts that describe objects, express quantity, and use specific prepositions, grammatical markers, and sentence structures that are age appropriate. The PLS-4 correlates with other standard measures of language development, with internal consistencies ranging from .67 to .88 for the subscales, and test re-test reliabilities ranging from .82 to .95 . Certification for all home visitors was similar to the WPPSI-III. The PLS-4 age standard scores were used for analysis purposes.

\section{Results}

\section{Analytic Strategy}

Analyses proceeded in three phases. First, the structure of the 10 indicators of cumulative (over the first three years of the study children's lives) chaos was examined using a combination of principle components (PCA) and exploratory factor analyses (EFA). Second, a series of regression models were estimated to test whether dimensions of household chaos made unique contributions to the prediction of two language outcomes (expressive and receptive language) that were measured when children were 36 months of age, above and beyond the effects attributable to child and primary caregiver covariates, including caregiver education and literacy level, household poverty (income to needs ratio), and observed parenting. Unless otherwise indicated, all of these covariates were averaged across visits. 
Given the natural metric of language outcomes (standard scores), unstandardized coefficients were reported (hence regression coefficients designate the change in standard score points that are associated with a one unit change in the predictor). All predictors were centered in order to ensure that the intercept was meaningful. Third, a path model was estimated to test whether the effects of chaos on child outcomes were mediated through dimensions of parenting. Statistical tests of the indirect effects provided a formal test of mediation.

Path analyses were estimated using full-information maximum-likelihood estimation, which assumed that data were missing at random. This provided a robustness check on results from regression models, which relied on listwise deletion and assumed that data were missing completely at random. Although listwise deletion methods are frequently criticized as the least optimal way for dealing with missing data, they are appropriate when the majority of missingness is on the dependent variable, as was the case here (Allison, 2001). Indeed, a majority of the missingness observed in this study resulted from those families who did not participate in the 36-month assessment, at which time child language was assessed. Among the $N=1,061$ children who completed both expressive and receptive language tests at the 36-month visit, $3.5 \%$ of children were missing data on temperament ratings, $1 \%$ were missing data on geographic isolation (primarily due to moves that took them out of the state), and less than one half of $1 \%$ were missing data on parenting composites. Complete data were available for all other predictors. This was due to the fact that most predictor variables were cumulative in nature and hence had values even when families missed one or more assessments. Regression models were estimated using Proc Surveyreg in SAS ${ }^{\circ}$ and the path model was estimated using Mplus version 5.1 (Muthén \& Muthén, 1998-2006), both of which accommodated the complex sampling design (i.e., stratification on income and race; individual probability weights associated with over-sampling of low-income and African American families).

\section{Structure of Cumulative Household Chaos}

In order to assess the first question, PCA was performed on the ten cumulative indicators of chaos. Bivariate correlations among the 10 cumulative indicators are summarized in Table 2. PCA indicated that two eigenvalues optimally represented the covariation in these 10 items. Following best practices, scree plots and parallel analyses were evaluated to determine the optimal number of factors to retain (Floyd \& Widaman, 1995; Dinno, 2009). Both methods favored a two-factor solution.

A follow-up EFA model was examined (see Table 3), which forced extraction of two correlated factors. We labeled the first factor Household Instability that included five variables: \# of people moving in and out of the household, the total number of people in the household, the number of household moves, the \# of changes in the primary caregiver, and the number of changes in the secondary caregiver. The second factor we labeled Household Disorganization, and it also included five variables: household density, the numbers of hours of TV watching, the preparation for home visits, the cleanliness of the home, and the neighborhood noise factors. Interestingly, these factors mapped almost completely onto the constructs identified as central to the definition of chaos (Evans \& Wachs, 2010). PCA and EFA results were consistent across weighted and unweighted analyses. These 10 indicators were standardized $(M=0, S D=1)$ and averaged to create two composite scores. The

Household Instability and Household Disorganization composites had reasonable internal consistency (Chronbach's alphas of .76 and .67, respectively) and were positively correlated with each other $(r=.38, p<.0001)$, as well as with parental education $\left(r_{\text {instability }}=-0.34\right.$; $\left.r_{\text {disorganization }}=-0.56, p \mathrm{~s}<.0001\right)$ and household income $\left(r_{\text {instability }}=-0.32 ; r_{\text {disorganization }}=\right.$ $-0.58, p s<.0001)$. 
Bivariate Correlations between Household Chaos, Child Language, and

Covariates-Bivariate correlations between child language scores, chaos composites, parenting composites, and the full set of covariates are provided in Table 4. Children's performance on expressive and receptive language tests was strongly correlated $(r=.68, p$ $<.001)$. Moreover, language scores were moderately correlated $(I \mathrm{~s}=.3-.4)$ with chaos disorganization (but not chaos instability), positive and negative parenting composites, and numerous household (income/needs ratio) and caregiver (age at the birth of her first child, education, literacy) covariates. Regression models provided a test of whether chaos composites were uniquely related to child language outcomes.

Prediction of Receptive Language-The first model regressed receptive language on all of the child, caregiver, and household covariates (hereafter referred to generically as covariates). This set of variables was predictive of receptive language $(F[13,991]=19.8, p$ $<.0001$, Adjusted $R^{2}=.23$ ). Child gender, caregiver age at the birth of their first child, caregiver literacy, and household income to needs were all significant predictors (see Table 5 for a synopsis of regression coefficients). Male children had lower receptive language scores than female children. Caregivers who were older at the time of birth of their first child, who performed better on the literacy screener, and who resided in higher income homes all had children with elevated receptive language scores.

The second model extended the first by including chaos composites (i.e., household instability and disorganization) as additional predictors. The set of variables continued to be predictive of receptive language $\left[F(15,991)=23.6, p<.0001\right.$, adjusted $\left.R^{2}=.26\right]$. The inclusion of household chaos composites explained an additional $3 \%$ of variation in receptive language scores beyond that attributable to covariates. Householddisorganization $(b=-6.6,95 \%$ confidence interval $[\mathrm{CI}]=-8.9---4.4, p<.0001)$, but not household instability $(b=1.6,95 \% \mathrm{CI}=-0.1-3.2, p=.06)$, was uniquely related to receptive language, even after taking into account a wide range of child, caregiver, and household variables that are known to be related to language development. Specifically, the more disorganized a child's home was across the first three years of life, the more poorly the child performed on a standardized assessment of receptive language. A follow up model that included squared terms for household disorganization and instability did not reveal any evidence for a nonlinear association between either chaos composite and receptive language (i.e., squared terms were not significant and are not reported).

The third model extended the second by including observed positive and negative parenting composites as additional predictors. The set of variables continued to predict expressive language $\left[F(17,991)=28.9, p<.0001\right.$, adjusted $\left.R^{2}=.29\right]$. The inclusion of parenting composites explained an additional $3 \%$ of variation in receptive language scores beyond that attributable to covariates and cumulative chaos. Inspection of regression coefficients indicated that both positive $(b=3.9,95 \% \mathrm{CI}=1.6-6.2, p=.0008)$ and negative $(b=-4.9$, $95 \% \mathrm{CI}=-7.3--2.6 p<.0001)$ parenting composites made unique contributions to receptive language. With the inclusion of parenting composites, the effect of chaos disorganization was reduced in magnitude but still statistically significant $(b=-5.1,95 \%$ confidence interval $[\mathrm{CI}]=-7.3--2.8, p<.0001$ ). This is consistent with, but not a definitive test of, the hypothesis that chaos exerts its effects on child language through its effect on observed parenting. A path analysis described below provided a formal test of mediation. Finally, with the inclusion of parenting composites, household instability emerged as a significant predictor $(b=1.8,95 \% \mathrm{CI}=0.2-3.5, p=.03)$, albeit in the opposite direction of disorganization. Greater instability was associated with slight improvements in receptive language. A synopsis of all regression coefficients for Receptive Language models is provided in Table 5. 
Prediction from Household Chaos: Expressive Language-The first model regressed expressive language on the set of covariates. This set of variables was predictive of expressive language $\left[F(13,1008)=16.8, p<.0001\right.$, Adjusted $\left.R^{2}=.21\right]$. Child gender, caregiver education, caregiver depression symptomatology, and caregiver literacy were all significant predictors (see Table 6 for a synopsis of regression coefficients). Male children had lower receptive language scores than female children. Caregivers who had higher education, who reported lower levels of depression symptoms, and who performed better on the literacy screener all had children with elevated expressive language scores.

The second model extended the first by including chaos composites (i.e., household instability and disorganization) as additional predictors. The set of variables continued to be predictive of expressive language $\left[F(15,1008)=19.6, p<.0001\right.$, adjusted $\left.R^{2}=.25\right]$. The inclusion of household chaos composites explained an additional $4 \%$ of variation in expressive language scores beyond that attributable to covariates. Householddisorganization $(b=-6.7,95 \% \mathrm{CI}=-9.0--4.4, p<.0001)$, but not household instability $(b=0.5,95 \% \mathrm{CI}$ $=-1.0-2.1, p=.50$ ), was uniquely related to expressive language, even after taking into account a wide range of child, caregiver, and household variables that are known to be related to language development. Specifically, the more disorganized a child's home was across the first three years of life, the more poorly the child performed on a standardized assessment of expressive language. A follow up model that included squared terms for household disorganization and instability did not reveal any evidence for a nonlinear association between either chaos composite and expressive language (i.e., squared terms were not significant and are not reported).

The third model extended the second by including observed positive and negative parenting composites as additional predictors. The set of variables continued to predict expressive language $\left[F(17,1008)=20.1, p<.0001\right.$, adjusted $\left.R^{2}=.26\right]$. The inclusion of parenting composites explained an additional $1 \%$ of variation in expressive language scores beyond that attributable to covariates and cumulative chaos. Inspection of regression coefficients indicated that both positive $(b=3.3,95 \% \mathrm{CI}=0.9-5.6, p=.007)$ and negative $(b=-3.2$, $95 \% \mathrm{CI}=-5.6--0.7, p=.01)$ parenting composites made unique contributions to expressive language. With the inclusion of parenting composites, the effect of chaos disorganization was reduced in magnitude but still statistically significant $(b=-5.6,95 \%$ confidence interval $[\mathrm{CI}]=-7.9--3.3, p<.0001$ ). This is consistent with, but not a definitive test of, the hypothesis that chaos exerts its effects on child language through its effect on observed parenting. A path analysis described below provided a formal test of mediation. A synopsis of all regression coefficients for expressive language models is provided in Table 6.

\section{Observed Parenting as a Mediator of the Association between Chaos and} Child Language-The previous regression models established that cumulative household chaos, measured across the first three years of life, was associated with child expressive and receptive language at 36 months of age, above and beyond the effects attributable to a large number of covariates. Moreover, the magnitude of this effect was reduced in the presence of observed parenting composites. A path model was estimated in order to formally test whether observed parenting mediated the association between household chaos and child language outcomes.

The path model was estimated using robust full-information maximum-likelihood estimation and included data from all 1,292 participants. The full set of predictors (covariates, chaos composites, parenting composites) explained approximately one fourth of the observed variation in language outcomes $\left(R_{\text {Expressive }}^{2}=.27, R_{\text {Receptive }}^{2}=.28\right)$. Moreover, covariates and chaos composites explained between one-fourth and one-half of the observed variation 
in parenting $\left(R^{2}\right.$ Positive Parenting $=.43, R^{2}$ Negative Parenting $\left.=.29\right)$. Consistent with results from regression models, there were significant direct effects from chaos disorganization to expressive $(b=-5.5, p<.001)$ and receptive $(b=-5.3, \mathrm{p}<.001)$ language. Moreover, there were significant indirect effects from chaos disorganization to expressive language through positive and negative (trend) parenting ( $b_{\text {disorganization }->>\text { positive }->>\text { expressive }}=-0.8, p=.01$; $b_{\text {disorganization --> negative --> expressive }}=-0.3, p=.06$ ), as well as significant indirect effects from chaos disorganization to receptive language through positive and negative parenting $\left(b_{\text {disorganization }->>\text { positive }-->\text { expressive }}=-0.9, p=.004 ; b_{\text {disorganization }->>\text { negative }->>\text { expressive }}=\right.$ $-0.5, p=.03)$. In contrast, none of the direct or indirect effects from household instability to child language scores were statistically significant (the direct effect from chaos instability to receptive language that was significant in regression model 3 was no longer significant: $b=$ $1.5, p=.08)$. Collectively, these results provide more definitive evidence that the effect of chaos disorganization on poorer language outcomes is partially mediated by observed parenting. A synopsis of standardized coefficients from the path model is provided in Figure 1. It is noteworthy that the parameter estimates from regression models were very similar in magnitude to those from path models (not presented). Indeed, none of the primary substantive conclusions differed across these two approaches. Hence, results were robust to assumptions about the missing data mechanism.

\section{Discussion}

The initial finding in this study was that our ten chaos indicators could be represented by two major factors: instability and disorganization. These two factors have recently been highlighted as the key constructs that define household chaos (Evans \& Wachs, 2010; Sameroff, 2010). Instability included changes in caregivers, moving households, and changes in people in the household over time, while disorganization included household density, household preparation for home visits, messiness of the house, and noise. Our study was the first to demonstrate that these factors could be extracted from 10 objective variables over children's first three years of life, without relying on parent subjective ratings, and to relate these to early language development in a representative sample of children in lowwealth communities.

The major findings from this study highlight the importance of the chaos dimension of disorganization as a proximal experience of young children in predicting early language development. Disorganization was a significant predictor of both receptive and expressive language, even in the presence of important covariates, such as family poverty, maternal literacy and depression, child gender and temperament, as well as geographic isolation. Even in the presence of observed parenting over three years that suggested partial mediation, disorganization still accounted for unique variance in predicting children's language. These findings support and extend previous work related to chaos and early language. For instance, Matheny et al. (1995) argued that two aspects of parent reported chaos from the CHAOS scale (household density and ambient noise) would be related to children's early language because of the overstimulation and subsequent withdrawal that children would experience by so many people in the home accompanied by ambient noise. They found a significant relationship between the two chaos composites and early poorer language in young children. In the current study, both household density and ambient noise loaded on disorganization which in turn predicted early language, suggesting that our cumulative more objective measure of disorganization was consistent with findings using the CHAOS scale, although our study found a stronger relationship and used a larger sample than previous studies. Our study also supported the recent findings in a sample of upper middle class families with twins (Johnson et al., 2008) that suggested that items from the CHAOS questionnaire that indexed "order" were related to children's language and literacy at school age. Our sample extends those findings for a diverse group of rural younger children. 
In the current study, disorganization was important even in the presence of poverty, suggesting that it is somewhat independent of poverty in predicting early language in this sample of mostly poor families (Bronfenbrenner \& Evans; Evans et al., 2005). In our sample, disorganization contributed considerably more variance in the prediction of both expressive and receptive language than either maternal education or poverty. Evans et al. (2005), in a sample of older children, found that chaos and poverty had almost completely shared variance in predicting social cognitive abilities, such that Evans argued that chaos was the more proximal cause of children's outcomes. Our study did not find this and is more supportive of Petrill et al. (2004) who found that chaos and SES contributed separate variance in predicting early language in children. Our study might be more in line with the Petrill et al. study because they, like the current study, examined young children who were 3 and/or 4 years of age. The Evans et al. study examined older children and had a different domain for child outcome. Thus chaos, and particularly disorganization, may have its greatest effect at early ages and in the area of language development.

Disorganization, in this study, may have been particularly predictive in comparison to some other studies because the construct was measured dynamically over the early years of life. Although the CHAOS questionnaire has clearly been a useful measure, the self report nature of the questionnaire, along with the fact that almost all studies used only one assessment point, may have limited its ability to account for larger amounts of the variance in predicting child and parenting outcomes. In the theoretical discussions of the role of chaos (Bronfenbrenner \& Evans, 2000; Evans, 2006), the impact of chaos was predicted to have its greatest impact when it was chronic so that children were experiencing chaos for long periods of time. We tried to capture this chaos over time by examining this construct over the first three years of life. In our more developmental and objective approach to the measurement of chaos, we used parent interview data of daily activities and changes in the household, along with home visitor reports at five points in time for most of our indicators over the child's first three years of life.

Even when both positive and negative parenting were entered into the regression, disorganization remained a significant predictor of language. Thus, this finding (Hart et al., 2007; Johnson et al., 2008; Petrill et al., 2004) suggested that parenting added about the same amount of variance as chaos in the prediction of both receptive and expressive language, but because of the decrease in the betas, there was a suggestion of mediation. In our path model, we formally tested the role of parenting as a mediator of child language. Collectively, the results provide more definitive evidence that the effect of chaos disorganization on poorer language outcomes is partially mediated by observed parenting. Thus, like the one other study to examine this three-part relationship (Evans et al., 1999), parenting only partially mediated the relationship between disorganization and child language. Interestingly, this previous study used household crowding as the measure of chaos. This variable in the current study (household density) loaded on the disorganization factor, suggesting the importance of household density and related factors in predicting early language.

Other studies have speculated on the role of parenting either by examining parenting in relationship to chaos or by discussing parenting as a future goal of research in this area (Corapci \& Wachs, 2002; Evans et al., 1999; Johnson et al., 2008; Matheny et al., 1995). These studies suggested that the overwhelming stress of chaos can diminish parents' ability to engage productively with their children. We supported this literature since positive and negative parenting, over a three-year period, partially mediated the association between disorganization and child language. Although some of the chaos indicators are less under the control of mothers, there are some possible ways mothers might lessen chaos in the home. For instance mothers could reduce the ambient noise in the home by reducing the number of 
hours the TV is on in the home. The average number of hours a day the TV was turned on was over four hours, even though $57 \%$ of the mothers were employed. In addition, mothers could try to lessen the effects of chaos on their own parenting by becoming more positive and sensitive in their interactions with their young children.

Although disorganization was clearly significant in our model, even in the presence of covariates and parenting, it accounted for only $3 \%$ to $4 \%$ of unique variance in child language. Although this may appear small, it would be a mistake to interpret the effect as small. The zero-order correlations give some sense of the effect sizes of each variable in the study on early language development (See Table 4). For instance, poverty (income/needs), which has been shown to be a major predictor of language development, and chaos seem equally related to child language with correlations around .40. It is likely that if income/ needs had been entered last into the regression, it would have accounted for a small amount of variance, just like chaos did. No other study of chaos has controlled for almost all important constructs that might be related to children's language including family variables (income, married, and geographical isolation), important maternal variables (age, education, employment, literacy level, and depression) and a host of child variables (age, gender, temperament, childcare settings, and child prenatal exposure to drugs/alcohol). Thus, it seems clear that disorganization is important as part of the family system of factors that should be considered in understanding children's early language development.

Although this study adds new and important information about the early correlates of chaos, poverty, and parenting on early language, there are a number of limitations to the study. We still need to understand whether these relationships among chaos, parenting, and language are maintained over time and whether there are particular periods in children's lives when chaos and other family processes are more or less consequential for children's development. There is also the possibility that household chaos might be related to other important early developmental skills that are not measured in this study. For instance, it might be particularly important to examine social/emotional development of young children and its relationship to chaos. Most of the previous work on instability of the household has speculated that changes in parents, homes, etc. have their greatest impact on both parent and child social/emotional adjustment. Adam (2004), in a review of studies examining instability, concluded that both the quality of the home and the instability of the home were related to parent and child social adjustment across childhood. More recent support for this notion is provided in a study by Marcynyszyn et al. (2008) that found that instability measures (changes in parent's intimate partners, residence, and children's schools) were related to depression and externalizing problems in adolescence, suggesting its influence on older children and in a different domain of development. Thus, it will be important for future studies to examine the relationship between these chaos constructs and other aspects of children's development, as well as to better understand how both constructs are related to development as the children get older. There are also some constructs related to chaos that were not measured in this study. For instance, family routines, including book-reading routines were not directly measured. Part of the reason they were not included in this study was because we would need to rely on parent report and because many routines, like sleep and eating schedules, are not stabilized for most young children until toddlerhood. Yet, these routines have been found to be important for children and have been used as measures in other chaos studies (Johnson et al., 2008; Roy et al., 2004). These routines would be important to measure in future studies and especially with older children.

In summary, this study contributes to our growing knowledge about the relationship between chaos and early development through an examination of the accumulated experience of chaos over early childhood in relationship to an important outcome, namely receptive and expressive language. Our findings suggest that daily household chaos (disorganization) over 
children's first three years of life may be one of the possible proximal pathways to children's poorer language development in low-wealth communities, as well as underscore the importance of this household disorganization in predicting child language, above and beyond the contribution of SES and parenting.

\section{Acknowledgments}

This research was supported by a grant from the National Institute of Child Health and Human Development (PO1HD-39667), Lynne-Vernon Feagans and Martha Cox, PIs, with co-funding from the National Institute on Drug Abuse and the Center for Minority Health. The Family Life Project Key Investigators include Lynne VernonFeagans, Martha Cox, Clancy Blair, Peg Burchinal, Linda Burton, Keith Crnic, Nan Crouter, Patricia GarrettPeters, Doug Granger, Mark Greenberg, Stephanie Lanza, Adele Miccio, Roger Mills-Koonce, Deborah Skinner, Cynthia Stifter, Emily Werner, and Mike Willoughby. We also want to thank all the families and children who participated in this longitudinal study.

\section{References}

Adam E. Beyond quality: Parental and residential stability and children's adjustment. Current Directions in Psychological Science. 2004; 13(5):210-213.

Aiken, LS.; West, SG. Multiple regression: Testing and interpreting interactions. Sage; Thousand Oaks, CA: 1991.

Arterberry ME, Midgett C, Putnick DL, Bornstein MH. Early attention and literacy experiences predict adaptive communication. First Language. 2007; 27(2):175-189.

Allison, PD. Missing data. Sage; Thousand Oaks, CA: 2001.

Bradley RH, Corwyn RF. Caring for children around the world: A view from HOME. International Journal of Behavioral Development. 2005; 29(6):468-478.

Brody GH, Flor D. Primary caregiver psychological functioning, family processes, and child adjustment in rural, single-parent, African-American families. Developmental Psychology. 1997; 33:1000-1011. [PubMed: 9383622]

Brody HB, Flor DL. Maternal resources, parenting practices, and child competence in rural, singleparent African American families. Child Development. 1998; 69(3):803-816. [PubMed: 9680686]

Bronfenbrenner U, Evans GW. Developmental science in the $21^{\text {st }}$ century: Emerging questions, theoretical models, research designs, and empirical findings. Social Development. 2000; 9(1):115125.

Brooks R, Meltzoff A. Infant gaze following and pointing predict accelerated vocabulary growth through two years of age: A longitudinal, growth curve modeling study. Journal of Child Language. 2008; 35:207-220. [PubMed: 18300435]

Brooks-Gunn J, Duncan GJ. The effects of poverty on children. The Future of Children. 1997; 7(2): 55-71. [PubMed: 9299837]

Burchinal M, Vernon-Feagans L, Cox MJ, The Key Family Life Project Investigators. Cumulative social risk, parenting and infant development in rural low-income communities. Parenting: Science and Practice. 2008; 8:41-69.

Coldwell J, Pike A, Dunn J. Household chaos-links with parenting and child behaviour. Journal of Child Psychology and Psychiatry. 2006; 47(11):1116-1122. [PubMed: 17076750]

Corapci F, Wachs TD. Does parental mood or efficacy mediate the influence of environmental chaos upon parenting behavior? Merrill-Palmer Quarterly. 2002; 48(2):182-201.

Derogatis, LR. The Brief Symptom Inventory (BSI): Administration, scoring and procedures manual. 4th Ed.. The Psychological Corporation; San Antonio, TX: 1993.

Derogatis, L. Brief Symptom Inventory 18. NCS Pearson; Minneapolis, MN: 2000.

Dill, BT.; Myers, SL. Rediscovering rural America. In: Blau, JR., editor. The Blackwell Companion to Sociology. Oxford, U.K.: 2008. p. 197-210.

Dinno A. Exploring the sensitivity of Horn's parallel analysis to the distributional form of random data. Multivariate Behavioral Research. 2009; 44(3):362-388. [PubMed: 20234802] 
Dodge K, Pettit G, Bates J. Socialization mediators of the relation between socioeconomic status and child conduct problems. Child Development. 1994; 65:649-665. [PubMed: 8013245]

Duncan, CM. Worlds apart: Why poverty persists in rural America. Yale University Press; New Haven, CT: 1999.

Edin, K.; Lein, L. Making ends meet: How single mothers survive welfare and low-wage work. Russell Sage; New York, NY: 1997.

Evans GW. Child development and the physical environment. Annual Review of Psychology. 2006; 57:423-451.

Evans GW, Gonnella C, Marcynyszyn LA, Gentile L, Salpekar N. The role of chaos in poverty and children's socioemotional adjustment. Psychological Science. 2005; 16(7):560-565. [PubMed: 16008790]

Evans, GW.; Kliewer, W.; Martin, J. The role of the physical environment in the health and well being of children. In: Schroeder, HE., editor. New directions in health psychology assessment. Hemisphere; New York, NY: 1991. p. 127-157.

Evans GW, Lepore SJ, Shejwal BR, Palsane MN. Chronic residential crowding and children's wellbeing: An ecological perspective. Child Development. 1998; 69:1514-1523. [PubMed: 9914637]

Evans GW, Maxwell LE, Hart B. Parental language and verbal responsiveness to children in crowded homes. Developmental Psychology. 1999; 35(4):1020-1023. [PubMed: 10442870]

Evans, G.; Wachs, T. Chaos and its influence on children's development: An ecological perspective. American Psychological Association; Washington, DC: 2010.

Floyd FJ, Widaman KF. Factor analysis in the development and refinement of clinical assessment instructions. Psychological Assessment. 1995; 7:286-299.

Garrett-Peters PT, Mills-Koonce WR, Adkins D, Vernon-Feagans L, Cox M, The Family Life Project Key Investigators. Early environmental correlates of maternal emotion talk. Parenting: Science and Practice. 2008; 8:117-152.

Garstein MA, Rothbart MK. Studying infant temperament via the revised infant behavior questionnaire. Infant Behavior and Development. 2003; 26:64-86.

Hane AA, Fox NA, Polak-Toste C, Ghera MM, Guner BM. Contextual basis of maternal perceptions of infant temperament. Developmental Psychology. 2006; 42:1077-1088. [PubMed: 17087543]

Hanson, T.; McLanahan, S.; Thomson, E. Economic resources, parental practices, and children's wellbeing. In: Duncan, G.; Brooks-Gunn, J., editors. Consequences of growing up poor. Sage; New York, NY: 1997. p. 190-238.

Hart SA, Petrill SA, Deater-Deckard K, Thompson LA. SES and CHAOS as environmental mediators of cognitive ability: A longitudinal genetic analysis. Intelligence. 2007; 33:233-242. [PubMed: 19319205]

Hart, B.; Risley, TR. Meaningful differences in the everyday experience of young American children. Brookes; Baltimore, MD: 1995.

Hoff E. The specificity of environmental influence: Socioeconomic status affects early vocabulary development via maternal speech. Child Development. 2003; 74:1368-1378. [PubMed: 14552403]

Hoff, E. Language development. 4th ed.. Wadsworth/Cengage Learning; Belmont, CA: 2009.

Johnson AD, Martin A, Brooks-Gunn J, Petrill SA. Order in the house! Associations among household chaos, the home literacy environment, maternal reading ability, and children's early reading. Merrill-Palmer Quarterly. 2008; 54(4):445-472. [PubMed: 19526070]

Kaufman, AS.; Kaufman, NL. KFAST: Kaufman Functional Academic Skills Test. PSYCAN; Toronto, Canada: 1994.

Lichter, DT.; Jensen, LI. Rural America in transition: Poverty and welfare at the turn of the $20^{\text {th }}$ century. In: Whitener, LA.; Weber, BA.; Duncan, G., editors. Rural dimensions of welfare reform. W.E. Upjohn Institute for Employment Research; Kalamazoo, MI: 2002. p. 77-112.

Marcynyszyn LA, Evans GW, Eckenrode J. Family instability during early and middle adolescence. Journal of Applied Developmental Psychology. 2008; 29(5):380-392.

Matheny A, Wachs TD, Ludwig J, Phillips K. Bringing order out of chaos: Psychometric characteristics of the Confusion, Hubbub, and Order Scale. Journal of Applied Developmental Psychology. 1995; 16:429-444. 
Maxwell LE. Home and school density effects on elementary school children: The role of spatial density. Environment and Behavior. 2003; 35:566-578.

Maxwell LM, Evans GW. The effects of noise on preschool children's prereading skills. Journal of Environmental Psychology. 2000; 20:91-97.

Muthén, LK.; Muthén, BO. Mplus User's Guide. Fourth Edition. Muthén \& Muthén; Los Angeles, CA: 1998-2006.

Milan S, Pinderhughes E. Family instability and child maladjustment trajectories during elementary school. Journal of Abnormal Child Psychology. 2006; 34(1):43-56. [PubMed: 16557358]

NICHD Early Child Care Research Network. Child care and mother-child interaction in the first three years of life. Developmental Psychology. 1999; 35(6):1399-1413. [PubMed: 10563730]

NICHD Early Child Care Research Network. The relation of child care to cognitive and language development. Child Development. 2000; 71(4):958-978.

O'Hare, WP.; Johnson, KM. Child poverty in rural America. Population Reference Bureau; Washington, D.C.: 2004. PRB Reports to America, Vol. 3, No. 1.

Pan BA, Rowe ML, Spier E, Tamis-Lemonda C. Measuring productive vocabulary of toddlers in lowincome families: Concurrent and predictive validity of three sources of data. Journal of Child Language. 2004; 31:587-608. [PubMed: 15612391]

Petrill SA, Pike A, Price T, Plomin R. Chaos in the home and socioeconomic status are associated with cognitive development in early childhood: Environmental mediators identified in a genetic design. Intelligence. 2004; 32:445-460.

Raviv T, Kessenich M, Morrison FJ. A mediational model of the association between socioeconomic status and three-year-old language abilities: The role of parenting factors. Early Childhood Research Quarterly. 2004; 19:528-547.

Reich W, Todd RD, Joyner CA, Neuman RJ, Heath AC. Reliability and stability of mothers' reports about their pregnancies with twins. Twin Research. 2003; 6(2):85-88. [PubMed: 12723994]

Rivers, K. Population Reference Bureau. Washington, D.C.: 2005. Rural Southern children falling behind in well-being; p. 1-3.

Roy KM, Tubbs CY, Burton LM. Don't have no time: Daily rhythms and the organization of time for low-income families. Family Relations. 2004; 53:168-178.

Sameroff, A. Dynamic developmental systems: Chaos and order. In: Evans, G.; Wachs, T., editors. Chaos and its influence on children's development: An ecological perspective. American Psychological Association; Washington, DC: 2010. p. 255-264.

Shipler, DK. The working poor: Invisible in America. Knopf; NY: 2004.

Tamis-LeMonda CS, Bornstein MH, Kahana-Kalman R, Baumwell L, Cyphers L. Predicting variation in the timing of linguistic milestones in the second year: An events-history approach. Journal of Child Language. 1998; 25:675-700. [PubMed: 10095330]

Tomasello M, Farrar MJ. Joint attention and early language. Child Development. 1986; 57:1454-1463. [PubMed: 3802971]

Tomasello M, Todd J. Joint attention and lexical acquisition style. First Language. 1983; 4(12):197_ 211.

Vernon-Feagans, L. Children's talk in communities and classrooms. Blackwell; Cambridge, MA: 1996.

Vernon-Feagans, L.; Gallagher, K.; Kainz, K. The transition to school in rural America: A focus on literacy. In: Eccles, J.; Meece, J., editors. Schooling and development. Erlbaum; Mahwah, NJ: in press

Wachs TD. Specificity of environmental action as manifest in environmental correlates of infant's mastery motivation. Developmental Psychology. 1987; 23(6):782-790.

Wachs TD. The nature of the physical microenvironment: An expanded classification system. Merrill Palmer Quarterly. 1989; 35:399-419.

Wachs TD, Chan A. Specificity of environmental action, as seen in environmental correlates of infants' communication performance. Child Development. 1986; 57:1464-1474.

Ward, S.; Turner, H. Work and welfare strategies among single mothers in rural New England: The role of social networks and social support. Paper presented at the Rural Poverty Workshop, Northeast Regional Center for Rural Development; Pennsylvania State University, PA. May. 2005 
Watt N, Wetherby AM, Shumway S. Prelinguistic predictors of language outcome at 3 years of age. Journal of Speech, Language and Hearing Research. 2006; 49:1224-1237.

Wechsler, D. Wechsler Preschool and Primary Scale of Intelligence-Third Edition (WPPSI-III). The Psychological Corporation; San Antonio, TX: 2003.

Westerlund M, Lagerberg D. Expressive vocabulary in 18-month-old children in relation to demographic factors, mother and child characteristics, communication style and shared reading. Child: Care, Health and Development. 2008; 34(2):257-266.

Zimmerman, L.; Steiner, VG.; Pond, RE. The Preschool Language Scale-IV. (PLS IV). Preschool Language Scale Fourth Edition: Examiner's Manual. The Psychological Corporation; San Antonio, TX: 2002. 


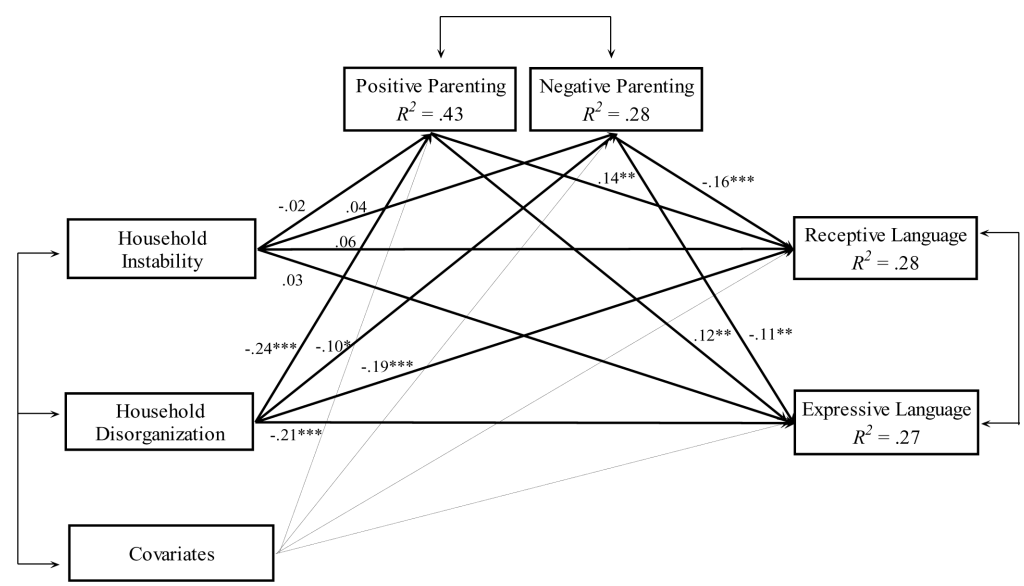

Figure 1.

Standardized regression coefficients from the path model relating household disorganization and instability to child language outcomes through observed parenting. 


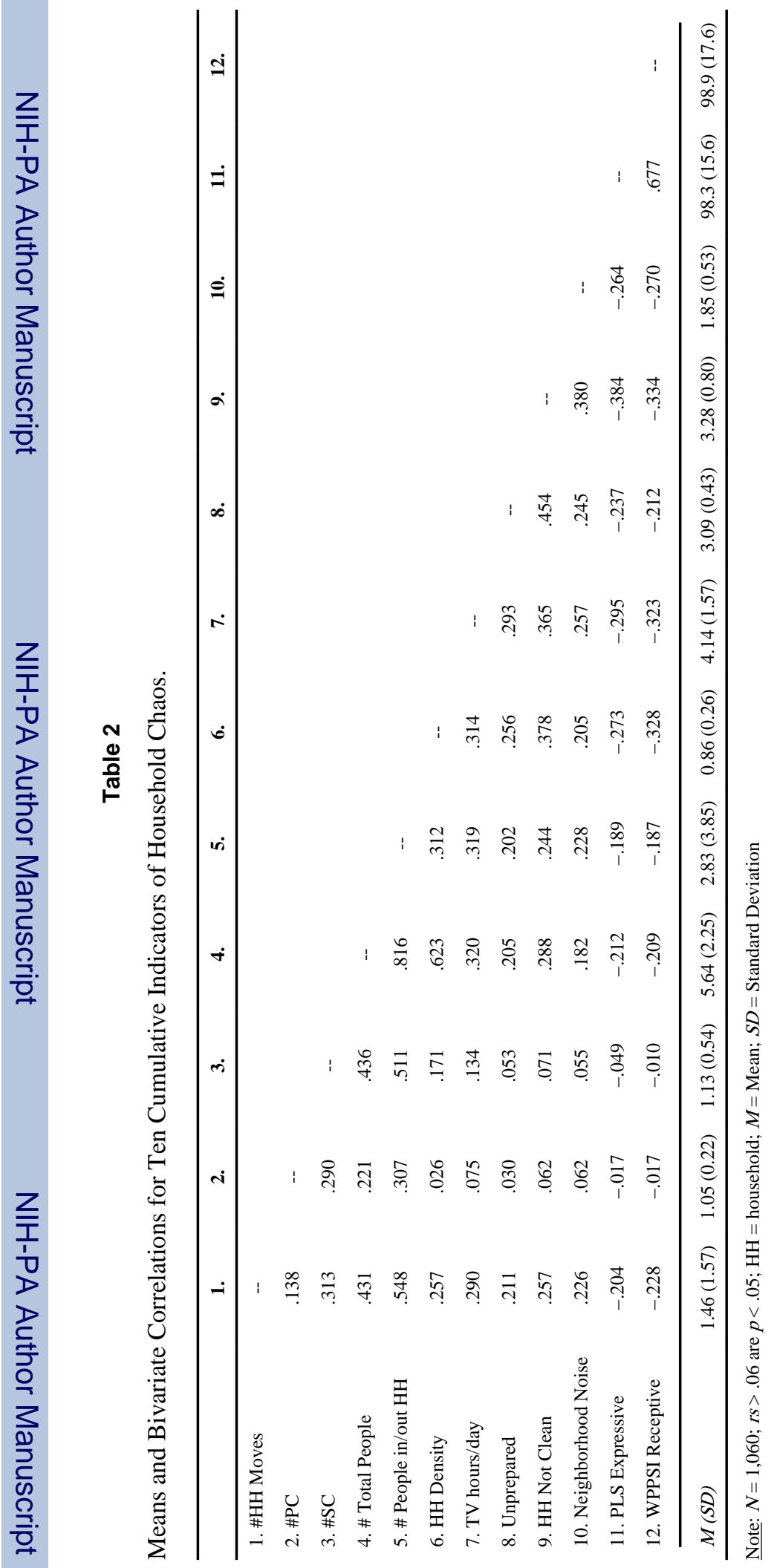

Early Child Res Q. Author manuscript; available in PMC 2013 July 01. 
Table 3

Factor Loadings from Exploratory Factor Analysis of Chaos Indicators

\begin{tabular}{lcc}
\hline & Factor I: Instability & Factor II: Disorganization \\
\cline { 2 - 3 } \# People in/out HH & $\mathbf{0 . 9 3}$ & 0.05 \\
\# Total People & $\mathbf{0 . 7 6}$ & 0.22 \\
\# HH Moves & $\mathbf{0 . 4 7}$ & 0.19 \\
\#PC & $\mathbf{0 . 3 8}$ & -0.10 \\
\#SC & $\mathbf{0 . 5 9}$ & -0.11 \\
\cline { 2 - 3 } HH Density & 0.12 & $\mathbf{0 . 5 3}$ \\
TV hours/day & 0.13 & $\mathbf{0 . 4 6}$ \\
Visit Preparation & 0.05 & $\mathbf{- 0 . 5 6}$ \\
HH Cleanliness & 0.09 & $\mathbf{- 0 . 7 4}$ \\
Neighborhood Noise & 0.03 & $\mathbf{0 . 4 4}$ \\
\hline
\end{tabular}

Note: $N=1,113$; inter-factor correlation $=.40 ; \mathrm{PC}=$ primary caresiver; $\mathrm{SC}=$ secondary caregivers; $\mathrm{HH}=$ Household; $\mathrm{TV}=$ television 


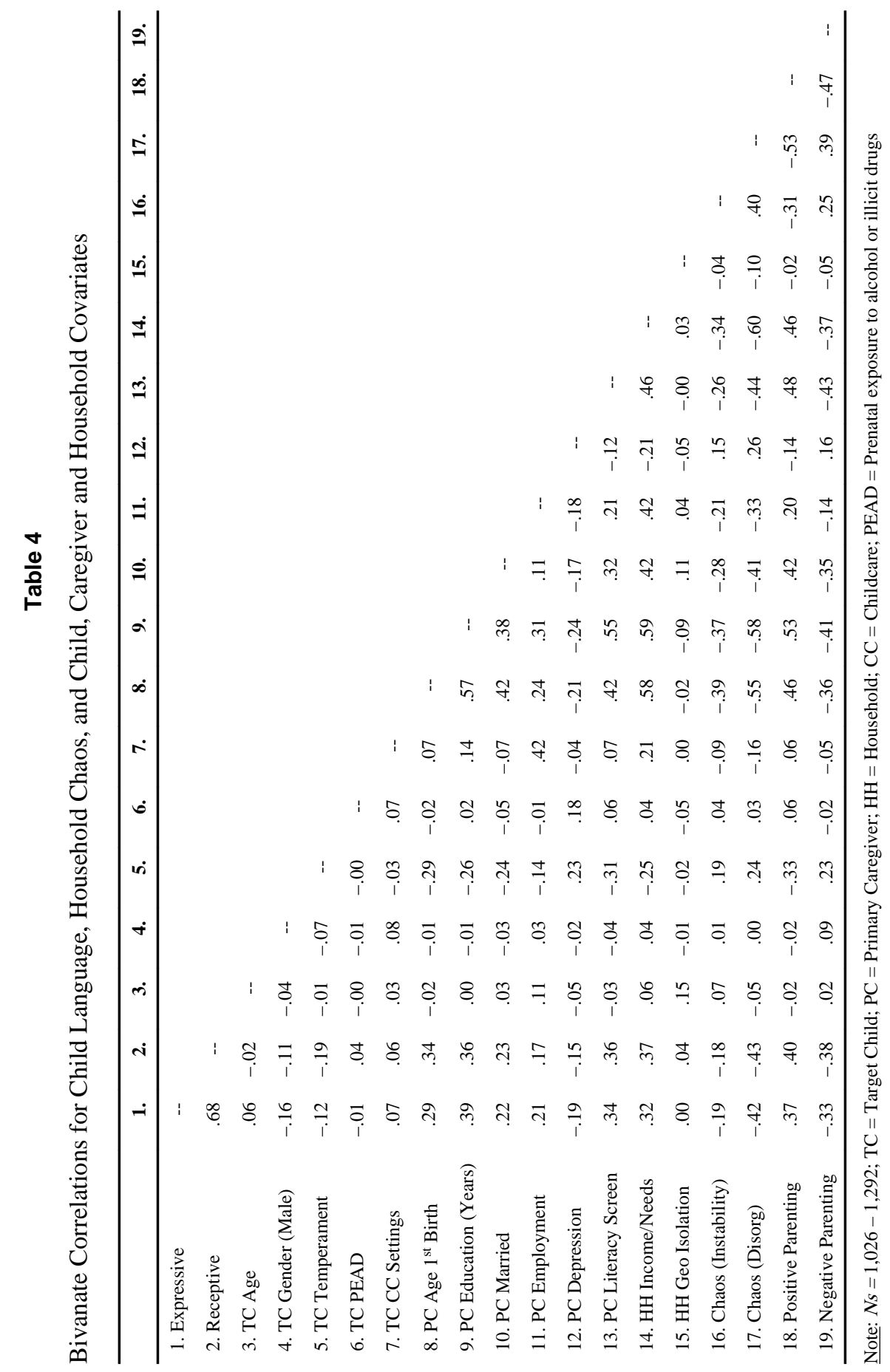

Early Child Res Q. Author manuscript; available in PMC 2013 July 01. 


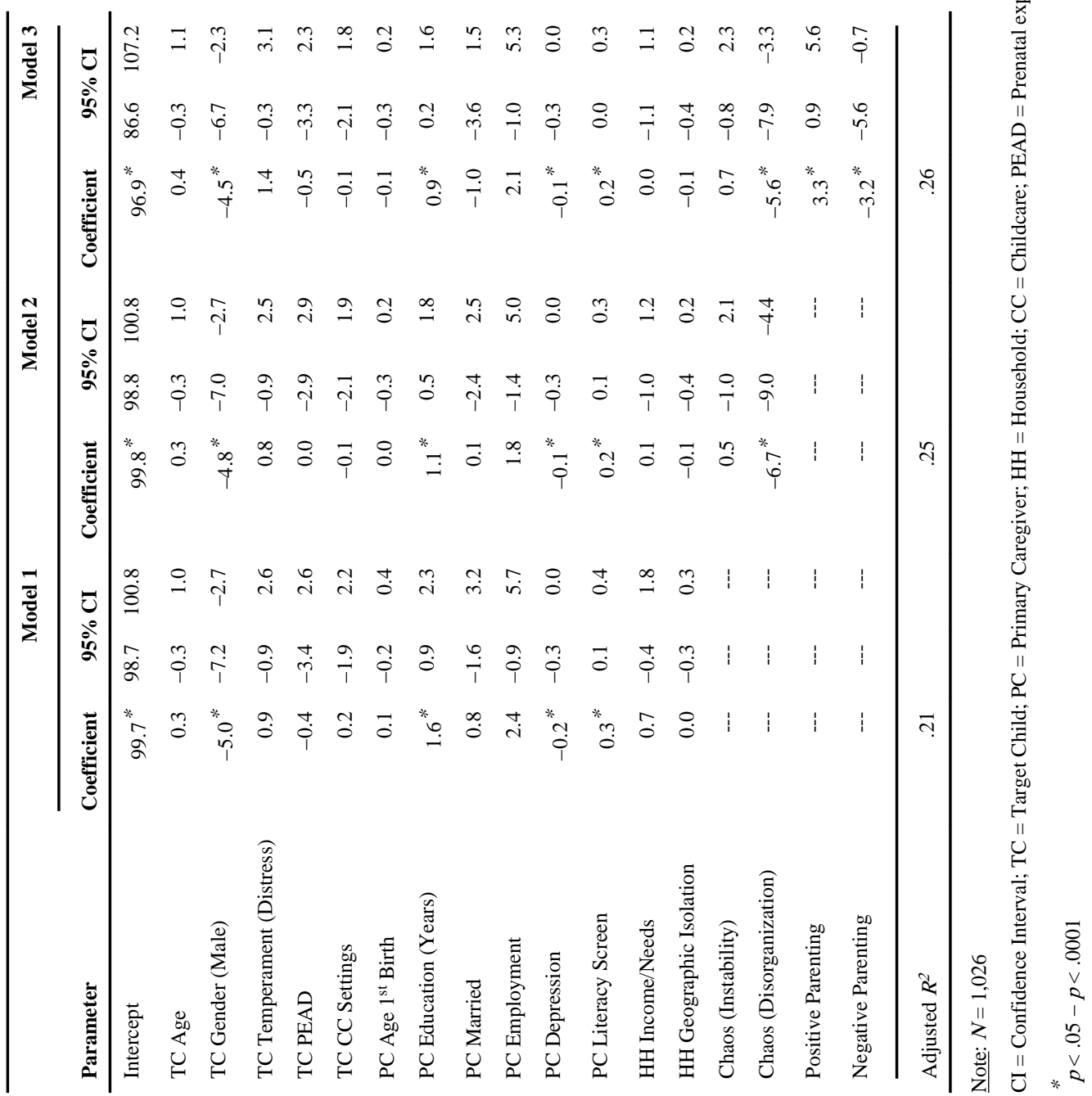

